\title{
Benchmarking Bimanual Cloth Manipulation
}

\author{
Irene Garcia-Camacho*1, Martina Lippi*2,3, Michael C. Welle ${ }^{2}$, Hang Yin $^{2}$, Rika Antonova ${ }^{2}$, Anastasiia Varava ${ }^{2}$, \\ Julia Borras ${ }^{1}$, Carme Torras ${ }^{1}$, Alessandro Marino ${ }^{4}$, Guillem Alenyà ${ }^{1}$, Danica Kragic ${ }^{2}$
}

\begin{abstract}
Cloth manipulation is a challenging task that, despite its importance, has received relatively little attention compared to rigid object manipulation. In this paper, we provide three benchmarks for evaluation and comparison of different approaches towards three basic tasks in cloth manipulation: spreading a tablecloth over a table, folding a towel, and dressing. The tasks can be executed on any bimanual robotic platform and the objects involved in the tasks are standardized and easy to acquire. We provide several complexity levels for each task, and describe the quality measures to evaluate task execution. Furthermore, we provide baseline solutions for all the tasks and evaluate them according to the proposed metrics.
\end{abstract}

Index Terms-Performance Evaluation and Benchmarking, Cooperating Robots

\section{INTRODUCTION}

$\mathbf{M}$ ANIPULATION of highly deformable objects, such as cloth, is an important area of robotics research that has applications both in industrial scenarios and in domestic environments. Despite its relevance, this research direction has historically received relatively little attention compared to rigid object manipulation due to the challenges it entails. Recently, a stronger interest in deformable object manipulation emerged and the survey in [1] presents the latest advances.

In order to effectively evaluate robotics methods, it is beneficial to provide specialized benchmarks [2]. A benchmark is a set of well-defined tasks to be performed in a standardized setup which needs to be easy to reproduce in different robotics laboratories. Existing manipulation benchmarks include large object sets [3], unified protocol procedures [4], robotic competitions [5], task specific benchmarks such as [6] for the picking task and also manipulation task taxonomies [7]. However, to the best of our knowledge, they all involve only rigid objects. In this paper, we provide benchmarks that will help assess the

Manuscript received: August, 15, 2019; Revised October, 19, 2019; Accepted December, 11, 2019. This paper was recommended for publication by Editor Han Ding upon evaluation of the Associate Editor and Reviewers' comments. This work receives funding from the European Union Horizon 2020 Programme under grant agreement no. 741930 (CLOTHILDE), Spanish State Research Agency through the BURG project (CHIST-ERA - PCIN2019103447) and the María de Maeztu Seal of Excellence to IRI (MDM-20160656), the "Ramon y Cajal" Fellowship RYC-2017-22703, the Knut and Alice Wallenberg Foundation, and the Swedish Research Council.

${ }^{1}$ Institut de Robòtica i Informàtica Industrial, CSIC-UPC, Barcelona, Spain \{igarcia, jborras, torras, galenya\}eiri.upc.edu

2 Robotics, Perception and Learning Lab, EECS at KTH Royal Institute of Technology Stockholm, Sweden \{lippi, mwelle, hyin, antonova, varava, dani\}ekth.se

3 University of Salerno, Salerno, Italy mlippidunisa. it

4 University of Cassino and Southern Lazio, Cassino, Italy alessandro.marinodunicas.it

*These authors contributed equally.

Digital Object Identifier (DOI): see top of this page. capability of a robotic system for manipulation of cloth-like objects.

To handle high degree of uncertainty about deformable objects' state, perception and manipulation often need to be intertwined. Furthermore, the choice of grasping and re-grasping strategies can significantly impact subsequent manipulation. Thus, one challenge in designing a benchmark for cloth manipulation is that different components of a robotic system, such as perception, grasping and manipulation planning, are highly dependent on each other. Therefore, we propose to evaluate the performance of the entire system rather than evaluating perception versus action components separately. We also recognize that for some tasks it is common to treat grasping of the initial target points as a sub-task. Hence, we provide a way to evaluate grasp execution, followed by evaluation of the task after the cloth is grasped.

We propose three benchmarks corresponding to three evaluation tasks that, in our opinion, form a basis for more complex tasks for handling clothes and dressing a human. (i) The first task is unfolding a tablecloth and spreading it over a table. The need to spread cloth-like objects, such as bed sheets and tablecloth, is ubiquitous in our everyday life. In addition, this task can be seen as preparatory, e.g spreading on a flat surface for ironing or folding. (ii) The second task is folding a towel on a table. This is one of the most common tasks in textile manipulation literature [8], and can be seen as a preparatory action before placing on a shelf or in a box for storage/packaging. Although prior works proposed methods for folding [9--13], these have never been systematically compared or benchmarked. (iii) The third task consists of fitting the neck of a T-shirt over a 3D printed head. This task is a simplification of a dressing scenario: a basis for more complex tasks like putting a T-shirt or a sweater on a human or mannequin.

We define performance metrics to evaluate a cloth manipulation method which are based on success of the task, execution time, force measures and, if possible, quality of the final result, e.g., we define how a tablecloth should be placed on the table. In this way, each proposed benchmark is well-equipped to distinguish approaches that are likely to perform general cloth manipulation well. Finally, we provide baseline solutions for all the tasks and evaluate them according to the proposed scoring, recording decreasing success rate as the complexity of scenarios increases.

Despite initial progress, cloth manipulation remains mostly unsolved, with innovative techniques still under development. Our proposed benchmarks form a systematic testbed for the prototypical cloth manipulation tasks, helping to evaluate 


\begin{tabular}{|c|c|c|}
\hline Code & \multicolumn{2}{|r|}{ Objects for manipulation } \\
\hline & Tablecloth & IKEA Fullkomlig $1.45 \times 2.4 \mathrm{~m}$ \\
\hline [st] & Small towel & IKEA towel Hären $0.3 \times 0.5 \mathrm{~m}$ or $0.3 \times 0.3 \mathrm{~m}$ \\
\hline & Big towel & IKEA towel Hären $0.5 \times 1 \mathrm{~m}$ or $0.4 \times 0.7 \mathrm{~m}$ \\
\hline & T-Shirt & Any T-shirt in accordance to Fig. 1 \\
\hline Code & \multicolumn{2}{|r|}{ Environmental objects } \\
\hline & \multirow{4}{*}{ Table } & Any table with dimension in the range \\
\hline & & Length: $[1.2,1.85] \mathrm{m}$ \\
\hline & & Width: $[0.7,0.8] \mathrm{m}$ \\
\hline & & Height: $[0.72,0.75] \mathrm{m}$ \\
\hline$[\mathbf{s}$ & Small head & Generate 3D model with provided script \\
\hline$[\mathrm{bh}]$ & Big head & Generate 3D model with provided script \\
\hline
\end{tabular}

TABLE I: List of objects with instructions for acquisition

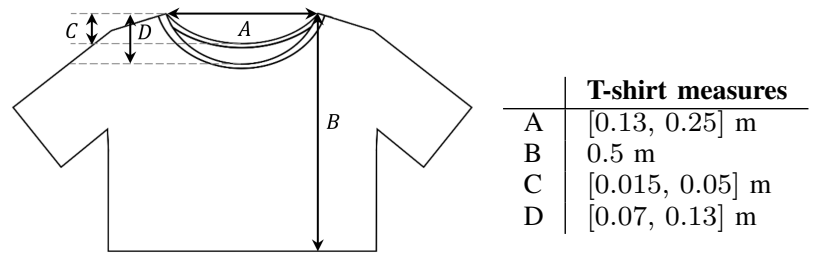

Fig. 1: Representation of the allowed measures for the T-shirt; B measure is fixed to equalize the level of difficulty when performing the dressing.

emerging approaches and to gain technical insights for further improvement.

\section{THE BENCHMARKS}

We propose three benchmarks for manipulation of highly deformable cloth-like objects which can be performed by any bimanual setup. In particular, our benchmarks focus on three basic tasks in cloth manipulation which involve textile objects of different sizes and types: spreading a tablecloth, folding a towel, and dressing. To foster easy modular use of the benchmarks, we separate each task into sub-tasks that can be evaluated individually, varying the level of difficulty on the basis of the cloth initial configuration and involved objects. Protocols (RAL-SI-2020-P19-0832_1-V1.0, RAL-SI2020-P19-0832_2-V1.0, RAL-SI-2020-P19-0832_3-V1.0 for the three tasks, respectively) can be found in the attached material and at the link with their respective explanatory videos and benchmark documents.

In the following we give a summary of the benchmarks tasks, setup and evaluation. For the sake of clearness, setup, sub-task decomposition and evaluation are common to three benchmarks and will thus be presented jointly. Further information can also be found on the website 1

\section{A. Tasks description}

1) Task 1: Spreading a tablecloth: This task consists of grasping a tablecloth and spreading it on a table, using the table and the tablecloth indicated in Table I. An example of implementation is shown in Fig. 2-left.

Similarly to the other tasks, this task requires to grasp the cloth at two grasping points, usually two of the corners, and then to manipulate it to spread it. For the first grasp, we

${ }^{1}$ https://ral-si.github.io/cloth-benchmark/ consider different starting cloth configurations: from folded to crumpled on the table (see Fig. 3). Grasping crumpled cloth at a desired grasping point has been attempted many times in literature by localizing corners or edges [9, 14] or more specific parts $[15-17]$. In contrast, starting from a folded configuration was rarely considered, despite it being a common cloth state in domestic environments. The challenge in this case lies in grasping just one single corner of the many layers that are folded together. After the first grasp, the cloth needs to be grasped at the second grasping point to unfold the cloth. Then it needs to be spread on the table. The task requires manipulating a big piece of cloth, which is challenging for many robots and may call for additional strategies. Overall, our protocol does not impose a specific strategy. This gives more freedom to researchers to develop and compare innovative approaches.

2) Task 2: Folding a towel: This task consists of grasping a towel and folding it. The task uses the same table as the previous task and two different sizes of towels, as indicated in Table I. An example of implementation is shown in Fig. 2 . middle. This is a classic cloth manipulation task. Since the early example of PR2 robot folding towels in 2010 [9], there have been many other works focusing on folding towels and other items [11, 12, 18,-20]. However, as stated in Section I] this task has never been benchmarked or properly compared based on the quality of the folds or execution time.

Folds location varies significantly depending on the garment geometry [13]. Even for a rectangular napkin or towel, there are multiple fold strategies one could follow. However, once the fold line has been decided, we want to focus on finding manipulation that can best realize it. For this reason, we focus on the simplest strategy for rectangular items: always fold in half and perform a maximum of three folds which we evaluate individually. This strategy has the advantage that it can be easily evaluated by taking top view snapshots after every fold. Besides the starting crumpled configuration (as in Task 1), we also consider a 'flat on the table' configuration, also shown in Fig. 3

When the cloth is crumpled, the main difference for grasping, compared to Task 1, is the size of the object. A small/medium versus large size would entail the need for different strategies to enable initial grasping. We do not impose a specific folding strategy. Small towels can be folded using the classic strategy of placing them on a table and picking two corners to fold [9, 12], but bigger ones might require alternatives [21].

3) Task 3: Partial dressing: The goal of the third task is to put a T-shirt over a simple head model starting from different initial configurations of the garment, as shown in Fig. 2-right. Putting on the sleeves is not included in the task. The complex geometric shape of T-shirts makes their manipulation towards desired states a difficult process that requires a tight integration of perceptive sensors, such as cameras and force/torque sensors, into the manipulation strategy. Thus, the focus of this task lies in evaluating the combination of perception and manipulation strategies.

Analogously to the previous tasks, the success of the manipulation task highly depends on the way the garment is 

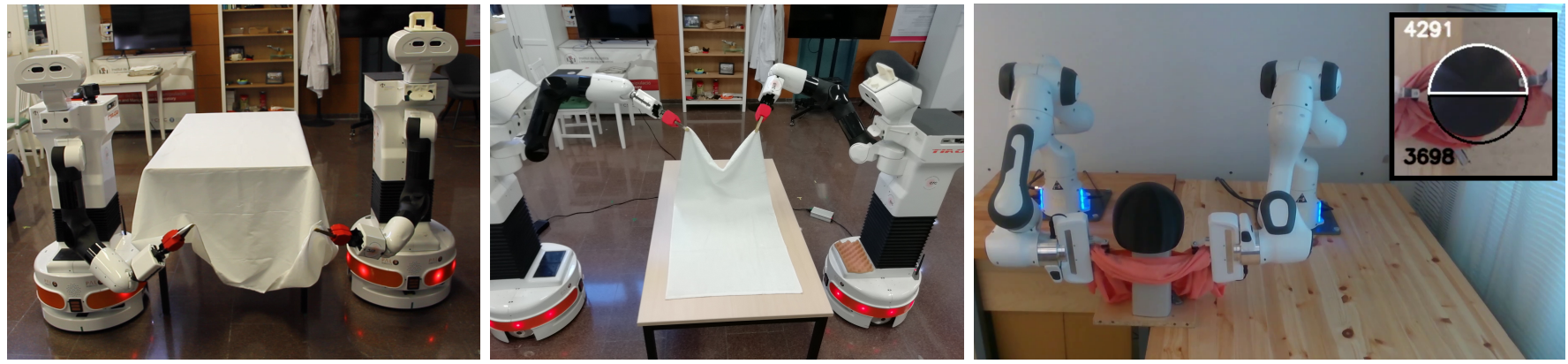

Fig. 2: From left to right, example of implementations of tasks one to three, respectively.

grasped, therefore we consider several initial configurations of the T-shirt which allow to explore different grasping strategies: crumpled, flat or folded on the table. Previous approaches to this task used reinforcement learning and topology coordinates [22, 23], but with a different starting configuration and assuming the cloth is pre-grasped.

Another important aspect is the relative size of the head with respect to the collar circumference: the larger the head is, the harder it is to execute the task. For this reason, we provide head models of two different sizes as reported in Table I. Finally, to avoid damaging the head and the garment, it is desirable to monitor applied forces especially when the collar is tight.

\section{B. Setup description}

1) Hardware description: Any bimanual setup with grasping capabilities can be employed and any sensor that can aid in completing the task is allowed (e.g., RGBD-cameras).

2) Objects description: Table I lists all the objects involved in the tasks with the link or information to acquire them. The YCB object set [4] includes two cloth items, that are a tablecloth and a T-shirt. The YCB tablecloth is designed to cover a standard $1.8 \mathrm{~m}$ long table until the floor. However, we propose a smaller IKEA tablecloth because its size is already challenging for the current state of the art. Regarding the YCB T-shirt, we observed how even T-shirts from the same batch have a high variance of measures. This also holds true for other available T-shirts. Therefore, we define a range of measures that are accepted for the T-shirt as reported in Figure 1. In this way, greater flexibility is guaranteed compared to the case of a predefined single T-shirt and the possibility of adopting the benchmark is maintained despite continuous changes in fashion. Any size from S to XL of the YCB T-shirt should fall into the allowed range. The length of the T-shirt (measure $\mathrm{B}$ in Figure 11) is fixed to allow comparability of different methods, since it determines the amount of garment that needs to pass through the head. Note that every T-shirt that is used needs to be measured even if it comes from the same batch to account for production variance. Finally, concerning the towel for Task 2, we include two sizes, a small towel ([st]) and a bigger one ([bt]). However, the sizes of the big and small towels slightly change depending on the country, therefore, we provide two options for the small towel and two for the big towel. Note that the use of big towels is already a step forward in the literature in terms of object size.

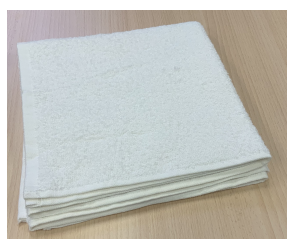

[fd]

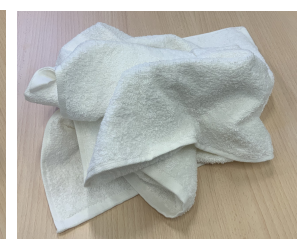

[cr]

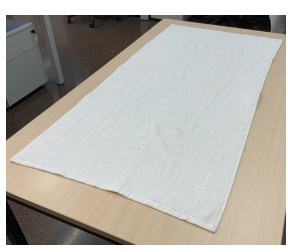

[ft]
Fig. 3: Examples of starting configurations with a towel: [fd]: folded on the left, [cr]: crumpled on the middle and [ft]: flat on the right.

In addition, two environmental objects are required, a table (for Tasks 1 and 2) and a human-like head (for Task 3). Following the idea of flexibility to make the setup easy to reproduce, we do not fix lightning conditions and we do not provide a specific table model but just an interval of table sizes. Concerning the human-like head, two different sizes are considered which are small ([sh]) and big ([bh]) and their models are defined according to the T-shirt measures. A script is provided in the attached material to automatically generate the 3D model. Refer to protocols for further details.

3) Initial cloth configuration descriptions: In general, when a task on cloth manipulation is attempted, the initial state of the cloth falls in one of these categories:

[pg2] Cloth is pre-grasped at two points.

[pg1] Cloth is pre-grasped at one point.

[ft] Cloth is lying flat on a table (Fig. 3-right).

[fd] Cloth is folded on a table (Fig. 3-left).

[cr] Cloth is crumpled on a table (Fig. 3 -middle).

These starting configurations will be common for all the protocols benchmarking each task, although not all starting configurations are used for all task. For instance, it is pointless to consider the folded configuration for the folding task.

We will refer to the parts of the cloth that need to be grasped as grasping points. In a towel, the grasping points are usually the corners but they can be redefined; instead, for a T-shirt, these strictly depend on the manipulation strategy.

\section{Sub-Tasks description}

Given a task, the respective sub-tasks are obtained by considering all the possible combinations of involved objects and initial cloth configurations: a tablecloth with 4 initial configurations for Task 1, two towels with 4 initial configurations for Task 2 and two head sizes with 5 initial configurations for Task 3. 
In addition, each sub-task can be decomposed in the following phases:

[GR1] Grasp first grasping point.

[GR2] Grasp second grasping point with other hand.

[MAN] Perform the manipulation (depending on the task). Note that both [GR1] and [GR2] may require manipulation; for instance, in order to grasp a crumpled cloth from a table and reach the first grasping point, the cloth may need to be premanipulated, and all this actions constitute the [GR1] phase. Obviously, no [GR1] and [GR2] phases are required in case of starting configuration [pg2] as well as no [GR1] phase is executed for the initial configuration [pg1].

Users can submit all phases of one sub-task, or just one phase alone. This subdivision in phases and sub-tasks allows to achieve incremental complexity, letting the user choose the desired level of difficulty to face, e.g., dressing task with small head and [pg2] initial configuration is clearly less challenging than the case of big head with [fd] initial configuration.

\section{Evaluation of results}

To enhance progress, allow reproducibility of results and easy comparison between different works, we propose the following list of performance metrics: success of each phase, execution time, force measures (if available) and quality measures. The choice not to provide a single value to assess goodness but a set of values is motivated by the fact that, in such complex tasks, the former may be too reductive; in this way, instead, each user can focus on the aspects of interest, e.g solutions that require longer time but exert lower forces. In the following, the proposed performance metrics are detailed.

1) Success of each phase: In light of the phases subdivision in Section II-C, each phase can be evaluated individually in regards to completeness. Phases [GR1] and [GR2] are considered successfully completed if the grasping is performed and is held during the whole manipulation and, in Tasks 1 and 3 , if the cloth is unfolded with starting configuration [fd]. The condition of success for phase [MAN] depends on the considered task: the tablecloth is successfully spread if it covers the table top; the folded towel is successfully folded if one fold is done and opposing corners are together (each fold is evaluated individually); for the dressing task we assume this phase is accomplished when the neck hole of the T-shirt is put over the head and the entirety of the T-shirt lies below the head. We let the users define different grasping points according to their strategy, not to limit the possible approaches in the [MAN] phase. In case the manipulation phase is successful, the grasping phases will be considered successful in turn.

To increase the flexibility of the benchmarks and promote participation, we leave the freedom to report only the manipulation part [MAN], which may be the case for end-to-end learning-based approaches, or only the grasping part [GR1] and [GR2] if a group is strong in grasping but lacks the perception solutions to successfully execute the manipulation. We believe this can be valuable to share solutions and combine different approaches to push the solutions forward.

2) Execution time: The execution time comprises the times needed for all the phases, and it is measured from the moment the first robot starts to move until the end of the manipulation.

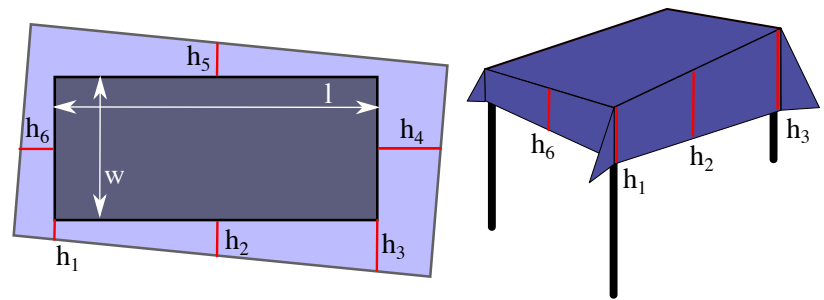

Fig. 4: Representation of the measures to evaluate how well the tablecloth has been placed.

3) Force measures: Force measures at the end effectors quantify the interaction between the robots and the environment; they are only acquired during phase [MAN] and minimum, maximum and average norms are considered. Note that, in order not to limit the possibility of using the benchmarks, force measures are not mandatory but are highly encouraged especially in dressing task, where monitoring of exerted forces on the head represents a key feature.

4) Quality measures: For the tasks of tablecloth spreading and folding, the quality of the result of the execution can be measured, e.g., poor results are achieved if the tablecloth is completely tilted or if towels are folded wrinkly or with the corners not matching.

To take that into account, we define a quality function that measures the percentage of error of the task result. Note that for the dressing task, no measures can be defined because of the binary nature of the task.

Quality measures for Task 1

We evaluate how much the tablecloth is rotated and translated with respect to the table. To this aim, as represented in Fig. 4 , a total of 6 tablecloth drop lengths at different sides of the table need to be measured after the tablecloth is spread. Measures can be taken from the middle of each table edge. For a table with length $t_{l}$ and width $t_{w}$ and a tablecloth with length $c_{l}$ and width $c_{w}$, the proposed percentage of errors are:

$$
\begin{aligned}
\% \text { rotation error: } E_{\alpha} & =\frac{\arctan \left(\frac{\left|h_{3}-h_{1}\right|}{t_{l}}\right)}{\pi / 4} \\
\% \text { length translation error: } E_{l} & =\frac{\left|h_{6}-h_{4}\right|}{c_{l}-t_{l}} \\
\% \text { width translation error: } E_{w} & =\frac{\left|h_{2}-h_{5}\right|}{c_{w}-t_{w}}
\end{aligned}
$$

These quality functions can only be applied if the task has been successfully accomplished, meaning that the tablecloth is covering all the table top. Then, a $100 \%$ rotation error occurs when the tablecloth is rotated by $\pi / 4$ radians $\left(45^{\circ}\right)$, which is unlikely to happen if the tablecloth is fully covering the table. The maximum translation error occurs when one of the hanging parts is zero, meaning the table is almost uncovered. If the hanging part of the table cloth is touching the floor, one needs to measure the tablecloth drop length ignoring the floor. If any circumstance occurs (e.g., one of the hanging parts of the tablecloth is wrinkled), one should report this with a picture, even if it does not affect the quality function. Note that this error measure is independent of the size of the table and 
tablecloth, thus allowing a fair comparison among different setups.

Quality measures for Task 2

We assume a one fold manipulation is successful if the corners of the original spread cloth are matching two by two. That means if one of the corners is bent, we assume the robot should correct that, otherwise the task cannot be reported as a success. In addition, we measure how well the corners match by evaluating the ratio between the surface of the spread cloth before and after the fold. Because our task is restricted to folding in half, each fold needs to cut in half the area of the spread cloth on the table. This has to be measured at each fold either by measuring manually the area or by automatically computing it with a top view image.

Then, the proposed quality function for this task is

$$
\% \text { of error in a fold } E_{f}=\frac{100}{0.5} \cdot\left\|\frac{A_{f}}{A_{i}}-0.5\right\|,
$$

where $A_{f}$ is the final area of the cloth from the top view, and $A_{i}$ is the initial area of the cloth. Assuming $A_{f}$ will always be smaller than $A_{i}, 100 \%$ error occurs when $A_{i}=A_{f}$, but also if $A_{f}$ is less than half of $A_{i}$, which can only happen if there are wrinkles or extra folds.

We promote the use of vision software to assess the area or the wrinkle state ${ }^{2}$ of the towel [24].

\section{E. Reporting results}

Based on the above, we require that, for each sub-task, five trials are performed and then, for each trial, measures II-D1. II-D4 are acquired. In addition, videos of the experiments and snapshots (or equivalent stylized figures) clearly representing the grasping points must be provided. A summary table, as shown in Table III. must be filled where, given a starting configuration, the success rate of each phase and average and variance of execution time, force measures and quality functions over the five trials are reported. When necessary, the size of the different elements must be reported as well, that are the table size for Tasks 1 and 2, the towel size for Task 2, and the head size for Task 3 . Note that, in the folding task, results associated with each fold must be provided and top view pictures of each fold state have to be reported. Moreover, in order to assess the generality of the proposed approach, it is required to specify which assumptions (in a set in the respective scoring sheet) are made for completing the task, e.g., knowledge of the cloth color and pattern. In the case new assumptions are considered with respect to those in the scoring sheets, a detailed description on how they affect the solution must be reported. Finally, a discussion on:

- Employed hardware/software setup with specification of robots' details and respective number of motors;

- What makes the system successful;

- What makes the system fail;

- What is improved compared to other methods;

should be provided. A thorough description for the scoring of each task can be found in the provided Benchmark documents 1

\footnotetext{
${ }^{2}$ https://gitlab.iri.upc.edu/labrobotica/algorithms/finddd_descriptor
}

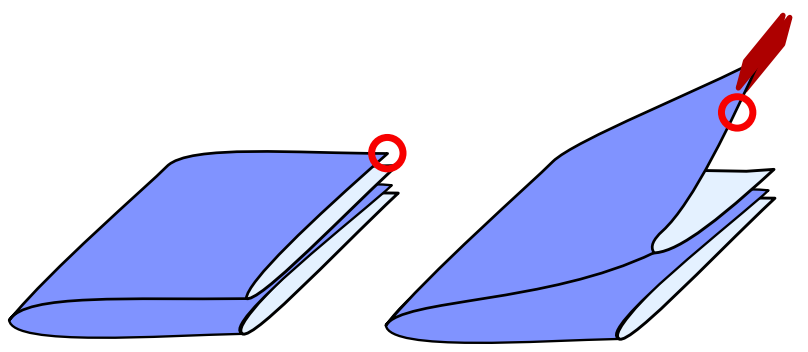

Fig. 5: The circles in red signal the location of the first grasping point for [GR1] (left) and on the initial grasp for the edge tracing in [GR2].

\section{BASELINE SYSTEMS}

To showcase how the presented Benchmarks should be used and promote comparison of different methods, we describe our own systems tackling the Benchmarks.

\section{A. Task 1: Spreading a tablecloth}

The robotic system used for the baseline solution for Task 1 (and also 2) is composed of two TIAGo robots (shown in Fig. 2-left). Because the tablecloth size is large, we can take advantage of the base mobility. The arms are equipped with a modified parallel gripper that is flexible when it touches the table to allow to safely contact the table before grasping but rigid in the grasped direction. A table with measures $1.20 \times$ $0.7 \times 0.73 \mathrm{~m}$ is used.

The solution for [GR1] to unfold the tablecloth is to grasp the first grasping point and pull the cloth up (Fig. 5-left). The solution for [GR2] is to grasp an edge point next to the first hand (Fig. 5-right) and trace the edge until the corner is reached. This implies sliding the cloth inside the gripper without loosing it. This manipulation has been previously applied only to very small clothes in [10], and there are some cloth specialized grippers designed to ease this manipulation [25]. Each grasp is performed by a different TIAGo robot, and after they have the tablecloth grasped, they move across the table to spread the tablecloth. Note that this strategy is applicable to different sized tables because bimanual manipulation is achieved with two independent robots.

Our method depends on some simplifying assumptions, reported in the Benchmark sheet. We assume the folded piece is oriented on the table so that the grasping point is the closest to the robot. However, the tablecloth can be placed anywhere on the table. The second grasp in [GR2], shown in Fig. 5. right, is assumed to be at a fixed position with respect to the hand that is already grasping. This holds true for most of the cases, but may fail when the cloth is twisted differently than expected. Finally, the robot knows the size of the cloth and the table, so that we can estimate beforehand the amount of displacement needed when both the robot tracks the edge and puts the tablecloth.

\section{B. Task 2: Folding a towel}

For the task of folding a towel, we use the same robotic system as in the previous task and we consider the big towel ([bt]) with measures $0.5 \times 1 \mathrm{~m}$. The two mobile manipulators 


\begin{tabular}{|c|c|c|c|c|c|}
\hline \multicolumn{7}{|c|}{ Task 1: Spreading a tablecloth } \\
\hline $\begin{array}{c}\text { Start. } \\
\text { Config. }\end{array}$ & $\begin{array}{c}\text { Mean quality funcs. } \\
\left(E_{\alpha}, E_{l}, E_{w}\right)\end{array}$ & $\begin{array}{c}\text { Success } \\
{[\text { [MAN] }}\end{array}$ & $\begin{array}{c}\text { Success } \\
\text { [GR2] }\end{array}$ & $\begin{array}{c}\text { Success } \\
\text { [GR1] }\end{array}$ & Time [s] \\
\hline [pg2] & $(1.44 \%, 8.23 \%, 17.67 \%)$ & $80 \%$ & & & 18.28 \\
\hline [pg1] & $(1.61 \%, 7.50 \%, 46 \%)$ & $60 \%$ & $80 \%$ & & 72.24 \\
\hline [cr] & - & $0 \%$ & $0 \%$ & $80 \%$ & - \\
\hline [fd] & - & $0 \%$ & $0 \%$ & $60 \%$ & - \\
\hline
\end{tabular}

\begin{tabular}{|c|c|c|c|c|c|}
\hline \multicolumn{6}{|c|}{ Task 2: Folding a towel } \\
\hline Towel size & \multicolumn{5}{|c|}{ [bt] } \\
\hline Fold & \multicolumn{5}{|c|}{ First fold } \\
\hline $\begin{array}{c}\text { Start. } \\
\text { Config. }\end{array}$ & $\begin{array}{c}\text { Success } \\
\text { [MAN] }\end{array}$ & $\begin{array}{c}\text { Success } \\
\text { [GR2] }\end{array}$ & $\begin{array}{c}\text { Success } \\
\text { [GR1] }\end{array}$ & $\begin{array}{c}\text { Mean quality } \\
\text { func. } E_{f}\end{array}$ & Time [s] \\
\hline [pg2] & $80 \%$ & & & $2.77 \%$ & 24.35 \\
\hline [pg1] & $\mathrm{np}$ & $\mathrm{np}$ & & $\mathrm{np}$ & $\mathrm{np}$ \\
\hline [cr] & $\mathrm{np}$ & $\mathrm{np}$ & $\mathrm{np}$ & $\mathrm{np}$ & $\mathrm{np}$ \\
\hline [ft] & $\mathrm{np}$ & $\mathrm{np}$ & $\mathrm{np}$ & $\mathrm{np}$ & $\mathrm{np}$ \\
\hline
\end{tabular}

\begin{tabular}{|c|c|c|c|c|c|c|c|c|c|c|c|c|c|c|}
\hline \multicolumn{15}{|c|}{ Task 3: Partial dressing } \\
\hline $\begin{array}{l}\text { Head Size } \\
\end{array}$ & \multicolumn{7}{|c|}{ [sh] } & \multicolumn{7}{|c|}{ [bh] } \\
\hline \multirow{2}{*}{$\begin{array}{c}\text { Start. } \\
\text { Config. }\end{array}$} & \multirow{2}{*}{$\begin{array}{l}\text { Success } \\
\text { [MAN] }\end{array}$} & \multirow{2}{*}{$\begin{array}{l}\text { Success } \\
\text { [GR2] }\end{array}$} & \multirow{2}{*}{$\begin{array}{l}\text { Success } \\
\text { [GR1] }\end{array}$} & \multirow{2}{*}{ Time [s] } & \multicolumn{3}{|c|}{ Force measures $[\mathrm{N}]$} & \multirow{2}{*}{$\begin{array}{l}\text { Success } \\
\text { [MAN] }\end{array}$} & \multirow{2}{*}{$\begin{array}{l}\text { Success } \\
\text { [GR2] }\end{array}$} & \multirow{2}{*}{$\begin{array}{l}\text { Success } \\
\text { [GR1] }\end{array}$} & \multirow{2}{*}{ Time $[\mathrm{s}]$} & \multicolumn{3}{|c|}{ Force measures $[\mathrm{N}]$} \\
\hline & & & & & $\min$ & avg & $\max$ & & & & & $\min$ & avg & $\max$ \\
\hline [pg2] & $100 \%$ & & & 29.30 & 0.54 & 3.02 & 7.29 & $100 \%$ & & & 76 & 0.38 & 2.94 & 7.46 \\
\hline [pg1] & $100 \%$ & $100 \%$ & & 48 & 0.49 & 3.89 & 9.34 & 80 & 10 & & 98 & 0.94 & 4.76 & 9.88 \\
\hline [cr] & $40 \%$ & $80 \%$ & $80 \%$ & 138.50 & 0.41 & 3.25 & 6.43 & $20 \%$ & $60 \%$ & $60 \%$ & 127.4 & 0.39 & 4.32 & 8.15 \\
\hline$[\mathrm{ft}]$ & $0 \%$ & $60 \%$ & $0 \%$ & - & - & - & - & $0 \%$ & $80 \%$ & $100 \%$ & - & - & - & - \\
\hline [fd] & $0 \%$ & $20 \%$ & $60 \%$ & - & - & - & - & $0 \%$ & $20 \%$ & $40 \%$ & - & - & - & - \\
\hline
\end{tabular}

TABLE II: Result summary tables. Notation "np" denotes that the respective sub-task has not been implemented in the baseline. For the sake of space, no variance values of execution times and forces are reported.

are placed at different sides of the table as shown in Fig. 2 . middle. We only report the [MAN] phase, as the other phases are similar to those in Task 1. Thus, we start with the two corners already grasped, and we then perform the folding motion. For the folding strategy, we focus on the first fold and we use a Dynamic Movement Primitives (DMP) representation of the motion for each robot learned by demonstration, and execute both trajectories in synchronization. The size of the towel and the localization of the robot with respect to the table are assumed to be known.

\section{Task 3: Partial dressing}

For the dressing task, we propose a human inspired solution based on a vision/force-feedback informed strategy with handtuned hyperparameters. All the possible head sizes described in II-B2 and starting configurations in III-B3 are considered. Moreover, experiments with two T-shirts are carried out to show the validity of the protocol as long as the T-shirt complies with the range of measures provided. In detail, the following set of measures $\{A, B, C, D\}$ hold for the two T-shirts, respectively: $\{0.19,0.5,0.029,0.1\} \mathrm{m}$ and $\{0.154,0.5,0.025,0.08\} \mathrm{m}$.

The robotic system, shown in Fig. 2-right, is composed of two Franka Emika Panda 7-DOFs manipulators equipped with parallel grippers. Customized long fingers have been adopted for one robot in order to have the fabric slipping into them during second grasping phase. The head is mounted on a podium stand in the middle of the workspace and its configuration is assumed to be known (in particular, its position is represented by the upper point along the axis of symmetry). Moreover, a Logitech USB camera is mounted over the setup to provide a bird eye view of the workspace and link-side torque sensors at each link of the robots are available; based on these, an estimate of the forces exerted at the end effector of each robot is given. For details on sensors and estimates accuracy, the reader can refer to [26].

Concerning the grasping phases [GR1] and [GR2], predefined grasping poses are selected with all the initial configurations, thus no visual feedback is exploited in these stages.
Concerning the manipulation phase [MAN], the formulation in [27] is leveraged for the dual-arm manipulation according to which the cooperative motion is expressed in terms of centroid and formation of the two end effectors. The basic idea of the devised strategy is to use the visual information to guide the team motion and, at the same time, to perform random wiggling motions which emulate human-like dressing. More specifically, the vision system splits the top-view circle associated with the head into two halves and measures the free area in each of them (see top right of Fig. 2-right). These measures are then exploited to determine in which direction to move the team centroid in such a way that both areas exceed a certain threshold. When the latter condition is fulfilled, the opening of the T-shirt is such that a sufficient surface of the head is visible through it, thus the downward motion to put on the T-shirt is started. In addition, small wiggling motions are introduced to facilitate the sliding of the garment along the head model. Finally, a continuous monitoring of end effector forces and of the elapsed time is performed: a restart procedure is planned when either force measurement exceeds a maximum allowed value or the elapsed time exceeds a time limit. For further details on the proposed baseline, the reader is referred to the document with solution comments in the accompanying material. Note that our baseline solution does not involve regrasping phases but these are generally allowed.

\section{RESUlts}

In this section, the evaluation of the baseline strategies according to the proposed Benchmarks is presented. Videos of each experiment and complete score sheets can be found in the results section at the website 1 A summary video is also provided in the accompanying multimedia material. For all the 3 tasks, performance results of the baseline solutions are shown in Table II

\section{A. Task 1: Spreading a tablecloth}

Regarding the manipulation ([pg2] row), the mobile base of the robots is very effective. Only occasional entanglements of 
the tablecloth cause strong forces and make the grippers loose the garment.

The second grasp ([pg1] row) is quite robust because the strategy of following the edge has proven to be effective: the first interest point is always at the same point under the other robot gripper, and the edge tracing takes advantage of the robot mobile torso to keep a vertical trajectory during as much time as possible. Failures are due to this last edge tracing phase: as the gripper has no force sensors, the edge is sometimes lost at the beginning of the movement.

Finally, in both [fd] and [cr] initial configurations, the first grasping phase resulted challenging. When [fd], because sometimes several layers are grasped causing the second grasp to fail. The image used to locate the corner is taken from the head of the robot, and the viewpoint and distance make difficult to localize a single garment layer. When [cr], because the friction of the fingers does not allow them to slide gently under the garment. In both cases, grasping fails and the task cannot be completed

\section{B. Task 2: Folding a towel}

Here we concentrate on the [MAN] itself and not on the [GR1] and [GR2] phases. Starting from initial configuration [pg2], [MAN] achieves quite a good success ratio of $80 \%$ and we only detected one problem in the final release step. In particular, in one of the trials our grippers, that have high friction fingertips, and the towel remained stuck until the arm moved away, creating an unwanted bending. As corners are not matching two and two, and our system is not able to correct this, we evaluated this trial as failure ${ }^{3}$

\section{Task 3: Partial dressing}

Performance results of the baseline solution with one T-shirt sample are summarized in Table III In detail, success rates for the different phases are reported in case of both small and big heads which make evident the increasing complexity and challenges introduced by the different combinations of starting configurations and head sizes. Indeed, different starting configurations lead to a wide variety of achievable grasping points but our manipulation strategy is only able to deal with a subset of them; in particular, our manipulation strategy assumes that the T-shirt is grasped at two points along the neck collar and that one side of the T-shirt is enclosed in the fingers in such a way to minimize the amount of fabric hanging under them. Indeed, most manipulation failures with starting configurations [ft] and [fd] are due to the fact that there is too much fabric below the grasping points and the robots are unable to find an opening of the T-shirt to pass it through the head. In addition, blind pre-defined grasping poses make manipulation in [cr] configuration generally challenging. Finally, grasping failures are recorded with starting configurations [fd] when several layers of the T-shirt are grasped with the first gripper and unfolding does not happen. Table II shows that the dressing task offers a wide range of possible improvements primarily

\footnotetext{
${ }^{3}$ The complete score sheets can be found at https://ral-si.github.io/ cloth-benchmark/\#results
}

in the manipulation phase but also in the grasping phases as each phase influences the following. For the sake of space, no further results are reported herein but complete scoring sheets and videos of the experiments are available at the link 3 for the other T-shirt sample as well. Finally, it is worth remarking that, even in cases with a $100 \%$ success rate, there is still a considerable margin for improvement with respect to execution times and exerted forces reported in Table III

\section{CONCLuSions}

In this paper, we proposed benchmarks for cloth manipulation with three representative tasks focusing on bimanual manipulation; they include cloth and garment items of various sizes. Each benchmark is hardware agnostic and flexible with respect to the strategies for solving the task. We believe that various robotics groups would find the benchmarks easy to use for comparing existing works and reporting new results. A simple well-defined object set and the possibility of reporting partial results make these benchmarks accessible to researchers targeting different stages of the tasks at various levels of difficulty. Our modular protocols also make the benchmarks potentially extendable to other tasks in the future.

We believe the baseline solutions give a valid initial point for comparison and show the increasing level of complexity of the different sub-tasks. Overall, this provides a good start for the research community to push the boundaries on what is possible in cloth manipulation further.

\section{REFERENCES}

[1] J. Sanchez, J.-A. Corrales, B.-C. Bouzgarrou, and Y. Mezouar, "Robotic manipulation and sensing of deformable objects in domestic and industrial applications: a survey," Int. J. Robot. Res., vol. 37, no. 7, pp. 688$716,2018$.

[2] F. Bonsignorio and A. P. del Pobil, "Toward replicable and measurable robotics research [from the guest editors]," IEEE Robot. Autom. Mag., vol. 22, no. 3, pp. 32-35, 2015.

[3] B. Calli, A. Singh, A. Walsman, S. Srinivasa, P. Abbeel, and A. M. Dollar, "The ycb object and model set: Towards common benchmarks for manipulation research," in Advanced Robotics (ICAR), 2015 Int. Conf. on, pp. 510-517, 2015.

[4] B. Calli, A. Walsman, A. Singh, S. Srinivasa, P. Abbeel, and A. M. Dollar, "Benchmarking in manipulation research: Using the yale-CMUberkeley object and model set," IEEE Robot. Autom. Mag., vol. 22, no. 3, pp. 36-52, 2015.

[5] F. Amigoni, E. Bastianelli, J. Berghofer, A. Bonarini, G. Fontana, N. Hochgeschwender, L. Iocchi, G. Kraetzschmar, P. Lima, M. Matteucci, P. Miraldo, D. Nardi, and V. Schiaffonati, "Competitions for benchmarking: Task and functionality scoring complete performance assessment," IEEE Robot. Autom. Mag., vol. 22, no. 3, pp. 53-61, 2015.

[6] J. Leitner, A. W. Tow, N. Sünderhauf, J. E. Dean, J. W. Durham, M. Cooper, M. Eich, C. Lehnert, R. Mangels, C. McCool, P. T. Kujala, and Lachlan, "The acrv picking benchmark: A robotic shelf picking benchmark to foster reproducible research," in IEEE Int. Conf. Robot. Autom., 2017.

[7] A. H. Quispe, H. B. Amor, and H. I. Christensen, "A taxonomy of benchmark tasks for robot manipulation," in Springer Proceedings in Advanced Robotics, pp. 405-421, Springer International Publishing, 2017.

[8] J. Borràs, G. Alenya, and C. Torras, "A grasping-centered analysis for cloth manipulation," arXiv:1906.08202, 2019.

[9] J. Maitin-Shepard, M. Cusumano-Towner, J. Lei, and P. Abbeel, "Cloth grasp point detection based on multiple-view geometric cues with application to robotic towel folding," in IEEE Int. Conf. Robot. Autom., pp. 2308-2315, 2010. 
[10] H. Yuba, S. Arnold, and K. Yamazaki, "Unfolding of a rectangular cloth from unarranged starting shapes by a dual-armed robot with a mechanism for managing recognition error and uncertainty," Adv. Robot., vol. 31, no. 10, pp. 544-556, 2017.

[11] A. Doumanoglou, J. Stria, G. Peleka, I. Mariolis, V. Petrik, A. Kargakos, L. Wagner, V. Hlavac, T.-K. Kim, and S. Malassiotis, "Folding clothes autonomously: A complete pipeline," IEEE Trans. Robot., vol. 32, no. 6, pp. 1461-1478, 2016.

[12] Y. Li, Y. Yue, D. Xu, E. Grinspun, and P. K. Allen, "Folding deformable objects using predictive simulation and trajectory optimization," in IEEE/RSJ Int. Conf. Intell. Robot. Syst., pp. 6000-6006, 2015.

[13] S. Miller, J. Van Den Berg, M. Fritz, T. Darrell, K. Goldberg, and P. Abbeel, "A geometric approach to robotic laundry folding," Int. J. Robot. Res., vol. 31, no. 2, 2012

[14] Y. Li, D. Xu, Y. Yue, Y. Wang, S.-F. Chang, E. Grinspun, and P. K. Allen, "Regrasping and unfolding of garments using predictive thin shell modeling," in IEEE Int. Conf. Robot. Autom., pp. 1382-1388, 2015.

[15] C. Bersch, B. Pitzer, and S. Kammel, "Bimanual robotic cloth manipulation for laundry folding," in IEEE/RSJ Int. Conf. Intell. Robot. Syst., pp. 1413-1419, 2011.

[16] A. Ramisa, G. Alenyà, F. Moreno-Noguer, and C. Torras, "Learning rgbd descriptors of garment parts for informed robot grasping," Engineering Applications of Artificial Intelligence, vol. 35, pp. 246-258, 2014.

[17] E. Corona, G. Alenyà, T. Gabas, and C. Torras, "Active garment recognition and target grasping point detection using deep learning," Pattern Recognition, vol. 74, pp. 629-641, 2018.

[18] M. Cusumano-Towner, A. Singh, S. Miller, J. F. O’Brien, and P. Abbeel, "Bringing clothing into desired configurations with limited perception," in IEEE Int. Conf. Robot. Autom., 2011.
[19] A. X. Lee, H. Lu, A. Gupta, S. Levine, and P. Abbeel, "Learning force-based manipulation of deformable objects from multiple demonstrations," in IEEE Int. Conf. Robot. Autom., 2015.

[20] J. Van Den Berg, S. Miller, K. Goldberg, and P. Abbeel, "Gravitybased robotic cloth folding," in Algorithmic Foundations of Robotics IX, pp. 409-424, Springer, 2010.

[21] K. Lakshmanan, A. Sachdev, Z. Xie, D. Berenson, K. Goldberg, and P. Abbeel, "A constraint-aware motion planning algorithm for robotic folding of clothes," in Experimental Robotics, pp. 547-562, 2013.

[22] T. Tamei, T. Matsubara, A. Rai, and T. Shibata, "Reinforcement learning of clothing assistance with a dual-arm robot," in IEEE-RAS Int. Conf. on Humanoid Robots, pp. 733-738, 2011.

[23] N. Koganti, T. Tamei, K. Ikeda, and T. Shibata, "Bayesian nonparametric learning of cloth models for real-time state estimation," IEEE Transactions on Robotics, vol. 33, no. 4, pp. 916-931, 2017.

[24] A. Ramisa, G. Alenya, F. Moreno-Noguer, and C. Torras, "Using depth and appearance features for informed robot grasping of highly wrinkled clothes," in IEEE Int. Conf. Robot. Autom., pp. 1703-1708, 2012.

[25] K. S. M. Sahari, H. Seki, Y. Kamiya, and M. Hikizu, "Edge tracing manipulation of clothes based on different gripper types," J. Comput. Sci., vol. 6, no. 8, pp. 872-879, 2010 .

[26] F. Ferraguti, A. Pertosa, C. Secchi, C. Fantuzzi, and M. Bonfè, "A methodology for comparative analysis of collaborative robots for industry 4.0," in Design, Automation Test in Europe Conference Exhibition, pp. 1070-1075, March 2019.

[27] P. Chiacchio, S. Chiaverini, and B. Siciliano, "Direct and inverse kinematics for coordinated motion tasks of a two-manipulator system," $J$. of dynamic systems, measurement, and control, vol. 118, no. 4, pp. 691697, 1996. 